\title{
Ultra-Compact Dwarf Galaxies in Galaxy Clusters
}

\author{
Michael J. Drinkwater ${ }^{\mathrm{A}, \mathrm{J}}$, Michael D. Gregg ${ }^{\mathrm{B}, \mathrm{C}}$, Warrick J. Couch ${ }^{\mathrm{D}}$, Henry C. Ferguson $^{\mathrm{E}}$, \\ Michael Hilker $^{\mathrm{F}}$, J. Bryn Jones ${ }^{\mathrm{G}}$, Arna Karick ${ }^{\mathrm{H}}$, and Steven Phillipps ${ }^{\mathrm{I}}$ \\ A Department of Physics, University of Queensland, Brisbane QLD 4072, Australia \\ B Department of Physics, University of California, Davis, CA 95616, USA \\ ${ }^{\mathrm{C}}$ Institute for Geophysics and Planetary Physics, Lawrence Livermore National Laboratory, L-413, \\ Livermore, CA 94550, USA \\ D School of Physics, University of New South Wales, Sydney NSW 2052, Australia \\ E Space Telescope Science Institute, 3700 San Martin Dve, Baltimore, MD 21218, USA \\ F Sternwarte der Universität Bonn, 53121 Bonn, Germany \\ G School of Physics and Astronomy, University of Nottingham, Nottingham NG7 2RD, UK \\ ${ }^{\mathrm{H}}$ School of Physics, University of Melbourne, Melbourne VIC 3010, Australia \\ I Astrophysics Group, Department of Physics, University of Bristol, Bristol BS8 1TL, UK \\ ${ }^{\mathrm{J}}$ Corresponding author. Email: mjd@physics.uq.edu.au
}

Received 2004 May 3, accepted 2004 September 27

\begin{abstract}
We review the evidence that the ultra-compact dwarf (UCD) galaxies we recently discovered in the Fornax Cluster form a new, previously unknown class of galaxies and we discuss possible scenarios for their formation. We then present recent results that UCDs are also present in the Virgo Cluster, and that there is a much larger than expected population of fainter UCDs in the Fornax Cluster. The size and properties of this population may lead us to revise our original 'galaxy threshing' hypothesis for the formation of UCDs.
\end{abstract}

Keywords: globular clusters: general — galaxies: dwarf — galaxies: formation — galaxies: clusters: general

\section{Introduction}

Dwarf galaxies have attracted increased attention in recent years, because of their recognised susceptibility to galaxy transformation processes within rich galaxy clusters (e.g. Bassino et al. 1994; Bekki et al. 2001; Moore 2003). Direct evidence for these processes, however, has been difficult to obtain, with a small number of diffuse light trails (Gregg \& West 1998) and intra-cluster stars (Durrell et al. 2002; Ford et al. 2002) being the only signs of galaxy disruption.

Our recent discovery of ultra-compact dwarf (UCD) galaxies may provide a new and easily measured tracer of disruptive processes in galaxy clusters if they prove to be the remnants of tidally disrupted nucleated dwarf elliptical (dE,N) galaxies as we have suggested (Bekki et al. 2001). If so, they might help explain the very large numbers of globular clusters associated with the central galaxies in clusters (Drinkwater et al. 2003) although new results presented here may lead to a revision of that model.

The Fornax Cluster Spectroscopic Survey (FCSS; Drinkwater et al. 2000a) is using the state-of-the-art TwoDegree Field (2dF) 400-fibre spectrograph on the AngloAustralian Telescope to obtain spectra for 14000 objects down to $B_{J}=19.8$ in a $4^{\circ} \times 3^{\circ}$ area centered on the Fornax Cluster. The survey is unique in targeting all objects, stellar and non-stellar selected from Schmidt plates in order to minimise selection biases. The most exciting find from the FCSS is the discovery of six objects from the unresolved 'stellar' targets which were actually cluster
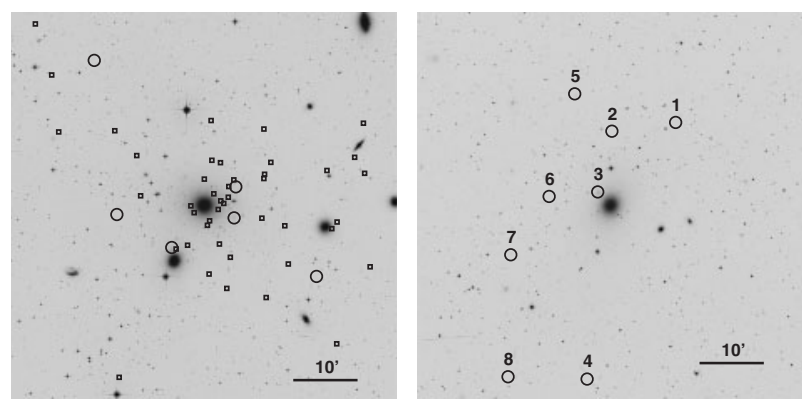

Figure 1 The locations of UCDs in the Fornax (left) and Virgo (right) Clusters. These sky survey images are both $1^{\circ}$ across, equivalent to $350 \mathrm{kpc}$ (Fornax at $20 \mathrm{Mpc}$ ) and $314 \mathrm{kpc}($ Virgo at $18 \mathrm{Mpc}$ ) respectively. In Fornax, the six original brighter UCDs are shown as circles, the new fainter UCDs as small squares. UCDs outnumber normal galaxies in this central part of the Fornax Cluster.

members with $-13<M_{B}<-11$, and sizes $\lesssim 100$ pc (Drinkwater et al. 2000b). We named these 'ultra-compact dwarf' (UCD) galaxies (Phillipps et al. 2001). These objects, shown in Figure 1, were passed over by early galaxy surveys because of their unresolved morphology, even though they are easily detected on photographic sky survey plates. Two were discovered prior to the FCSS with CCD observations (Hilker et al. 1999).

In this paper we review the nature of UCDs (Section 2) and present our discovery of new UCD populations (Section 3). We conclude with a summary of our current understanding of these curious objects (Section 4). 


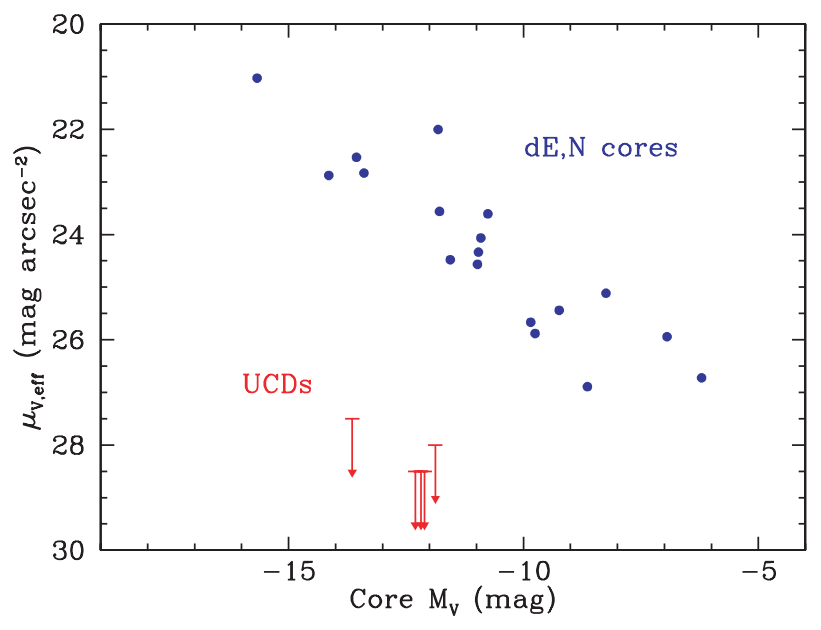

Figure 2 Comparison of the morphology of UCDs with normal nucleated dwarf ellipticals in Fornax. The effective envelope surface brightness in $V$, measured in fixed apertures excluding the cores, is plotted as a function of the $V$-band absolute magnitude of the cores. The surface brightness of the UCDs are upper limits as no envelopes were detected on scales greater than the image resolution ( 2 arcsec full-width half-maximum). The data are from deep images of $2.4 \mathrm{deg}^{2}$ in the centre of the Fornax Cluster taken with the $100^{\prime \prime}$ du Pont telescope of the Las Campanas Observatory.

\section{The Nature of UCDs}

From our original observations we were unable to determine if the Fornax UCDs were over-luminous, intracluster globular clusters, extreme examples of the normal dwarf galaxy population, or some entirely new class of object. Deep CCD imaging of the the Fornax Cluster (Hilker et al. 2003) has established that the UCDs are not an extreme tail of the normal dwarf galaxy population; see Figure 2. The figure shows that the UCD fall over four magnitudes below the dwarf-galaxy relationship between core luminosity and envelope surface brightness. (Hubble Space Telescope (HST) imaging indicates that dwarf galaxy cores are similar to UCDs in surface brightness and luminosity.) Due to the all-object nature of the FCSS we know that galaxies with intermediate properties between the UCDs and the normal galaxies do not exist in the cluster. If UCDs form by tidal stripping, this would imply a relatively short timescale given the lack of partially stripped objects. The survival of the remaining dwarf galaxies would then be explained by their lying on higher-energy orbits that do not pass close enough to the cluster centre for significant threshing to take place.

We obtained HST images of five UCDs in Cycle 9. They were all resolved, with effective radii of $10-22 \mathrm{pc}$, much larger than the biggest globular clusters $(5 \mathrm{pc})$, but similar in size to dwarf galaxy nuclei; see Figure 3. We also obtained high resolution VLT and Keck spectra of the objects to measure velocity dispersions: these are plotted against luminosity in Figure 4A, along with bright ellipticals, dEs, and M31/Galactic globular clusters. The bright galaxies follow the Faber-Jackson (1976) $L \propto \sigma^{4}$ relation, while the globular clusters lie along a different locus with $L \propto \sigma^{1.7}$ (Djorgovski et al. 1997). The UCDs fall along

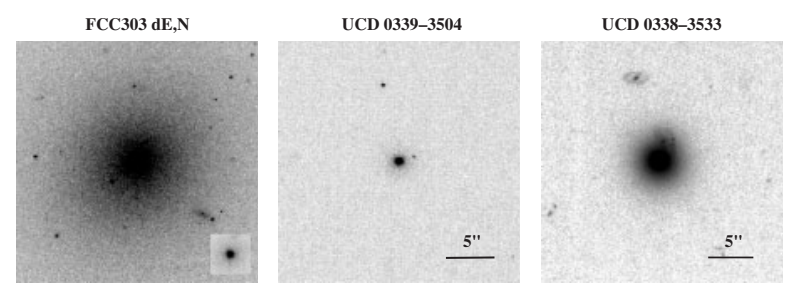

Figure 3 Comparison HST/STIS broad band images of a Fornax nucleated dwarf (left) and a mid-size Fornax UCD (centre). The dE halo more than fills the 50 arcsec field; its nucleus is shown at a different stretch in the inset to underscore the similarity with the UCD. The largest UCD (right, enlarged compared to the other two images) has a small exponential halo surrounding the core. Note the compact structures surrounded by a faint envelope to the northwest.

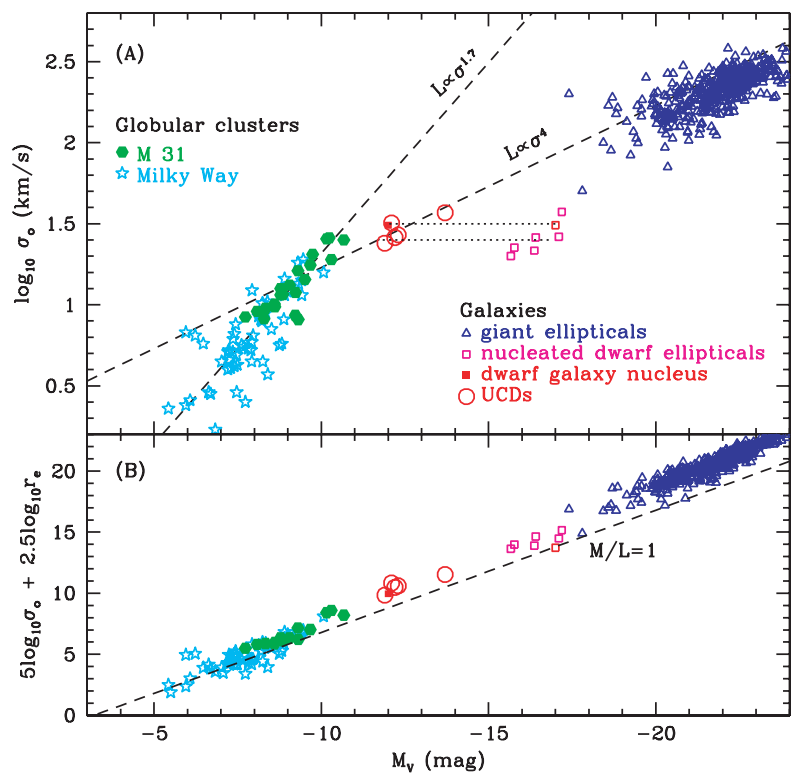

Figure 4 Comparison of UCDs with normal galaxies and globular clusters. (A) Internal velocity dispersion versus luminosity. Dashed lines: the Faber-Jackson relation for elliptical galaxies and the steeper relation followed by globular clusters. Dotted lines: the path predicted by our 'threshing' scenario for nucleated dwarfs stripped to form UCDs. The properties of the dwarf galaxy nuclei were obtained by model subtracting their envelopes from our HST images. (B) A combination of velocity dispersion and radius equivalent to mass plotted against luminosity. Dashed line: a constant mass-to-light ratio of unity.

the extrapolated Faber-Jackson relation, but are an order of magnitude too bright for their dispersions to fall on the globular cluster relation. Combining HST-measured radii with velocity dispersions, we estimate the UCD masses (see Figure 4B). They have $V$-band mass-to-light ratios of $M / L=2-4$ in Solar units, higher than the most massive M31 globular clusters $(M / L=1-2)$. These results show that UCDs are distinct from both globular clusters and normal dwarf galaxies - a new class of galaxy (Drinkwater et al. 2003). Our preferred model for their formation is by 'galaxy threshing' (Bekki et al. 2001), tidal encounters with the central galaxy in which nucleated dwarf galaxies lose their envelopes ( $\sim 98 \%$ of their original luminosity) while the nuclei are little affected, as shown in Figure 4A. 


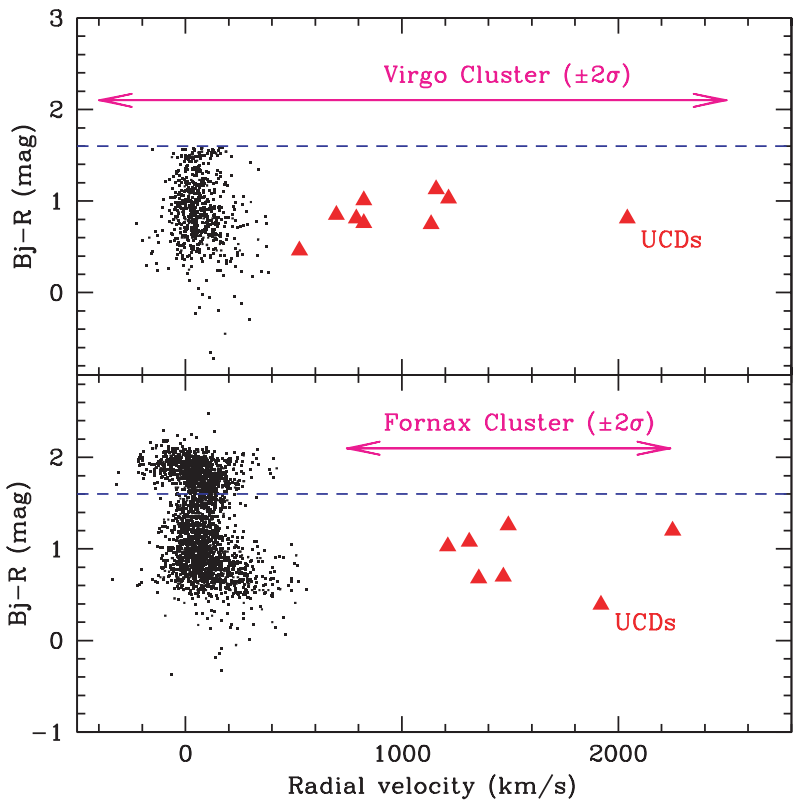

Figure 5 Colour-velocity distributions of stars and UCDs in the Fornax and Virgo fields. The lower panel shows the photographic $B_{J}-R$ colours of the stars (black dots) and UCDs (red triangles) observed in the Fornax survey, along with the $2 \sigma$ velocity range of cluster galaxies. The upper panel shows the same parameters for the Virgo survey, where we only observed targets with $B_{J}-R<1.6$.

\section{New UCD Populations}

If, as we proposed, UCDs are formed by 'galaxy threshing' (Bekki et al. 2001) of nucleated dwarf elliptical galaxies, then they should be found in similar numbers in the centre of the Virgo Cluster. This is because the central region of Virgo (see Figure 1) contains a similar-sized galaxy (M87) to NGC 1399 in Fornax, and a very similar surface density of $\mathrm{dE}, \mathrm{N}$ galaxies. To test this prediction, we undertook similar $2 \mathrm{dF}$ spectroscopy in Virgo. In this case, informed by the properties of the Fornax objects, we targeted a restricted colour range of unresolved objects within $0.5^{\circ}$ of M87. As shown in the lower panel of Figure 5, the Fornax UCDs are all bluer than $B_{J}-R=1.4$, so in Virgo we only observed targets with $B_{J}-R<1.6$. With just three hours of $2 \mathrm{dF}$ service observing we measured 1000 objects and readily detected eight Virgo UCDs within 45 to $200 \mathrm{kpc}$ of M87 (shown in Figure 1). This number of UCDs is consistent with the same UCD surface density as in the Fornax Cluster. This suggests that UCDs may be a common population in galaxy clusters.

If UCDs are indeed the remnant nuclei of disrupted $\mathrm{dE}, \mathrm{N}$ galaxies, they should, to first order, have a similar luminosity function to that of the nuclei of the parent dwarf galaxies which peaks some two magnitudes fainter than the limit of our discovery $2 \mathrm{dF}$ observations (see Figure 6). A clear prediction of our threshing hypothesis is that the original UCDs are just the high-luminosity tip of the distribution. In recent (2003 October) $2 \mathrm{dF}$ observations we extended our UCD search 1.5 mag fainter and found an additional 46 objects, shown in Figure 1, giving a total of 52 compact objects in the cluster. Observations to fainter

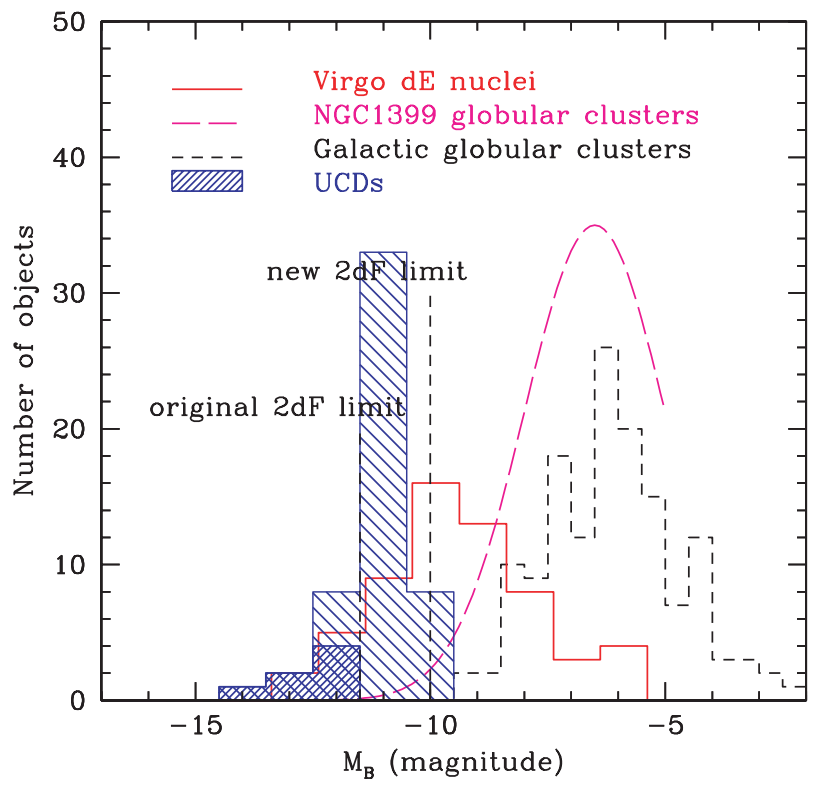

Figure 6 Absolute magnitude distribution of the UCDs compared to other compact systems. The original UCD population (dark shaded histogram) and the new fainter sample (light shaded histogram) are shown. The comparison populations are $\mathrm{dE}, \mathrm{N}$ galaxies in the Virgo Cluster (Binggeli \& Cameron 1991; solid line), a model fit to the globular clusters around NGC 1399 (Bridges, Hanes, \& Harris 1991; long dashes) and Galactic globular clusters (Harris 1996; short dashes). Note: the magnitude limit of our survey that found the compact objects corresponds to $M_{B}=-11$.

limits by Mieske et al. $(2002,2004)$ in the very centre of the cluster have detected similar numbers of even less luminous compact objects. The UCDs are so numerous that they outnumber all normal galaxies in this central region of the cluster; this implies a larger population of precursor objects than originally assumed in our galaxy threshing (Bekki et al. 2003) scenario which may now need revising.

\section{Discussion and Summary}

Our follow-up observations of our original discovery of of UCDs have established that they represent a new type of galaxy, only the second new class to be found (after QSOs) since the discovery of dwarf spheroidal galaxies by Shapley (1938). The internal properties of the UCDs, as measured by high-resolution spectroscopy and imaging, are consistent with our 'galaxy threshing' hypothesis that they are the residual cores of nucleated dwarf elliptical galaxies tidally disrupted in the cluster potential. This hypothesis is further supported by our discovery of a second population of UCDs in the Virgo Clusters.

The existence of UCDs places new constraints of theories of galaxy formation, notably the distribution of dark matter in dwarf galaxies. Assuming that the UCDs formed by threshing, they provide evidence against the presence of very cuspy dark matter halos, as these would make the dwarfs stable against threshing (Moore 2003).

However, the large population of fainter UCDs we have discovered in the Fornax Cluster may lead to a revision of our threshing hypothesis, because it implies even more 


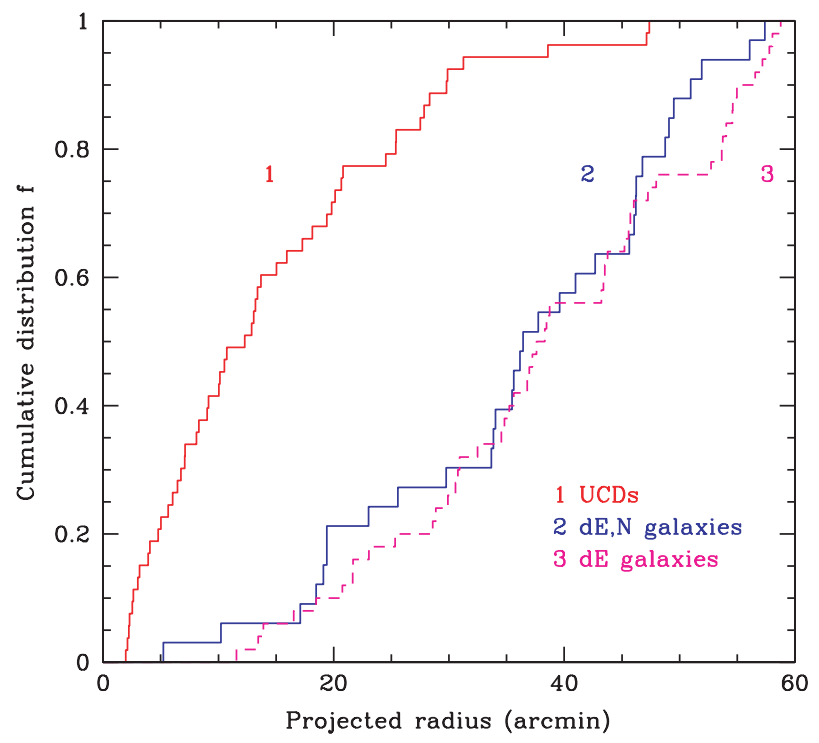

Figure 7 Cumulative radial distribution of UCDs in the Fornax Cluster compared to nucleated (dE,N) and normal (dE) dwarf galaxies. For each population the cumulative distribution is plotted as a function of projected radius within the 60 arcmin limit within which we have a complete sample of UCDs.

galaxies were threshed than in our simulations (e.g. Bekki et al. 2003). A particular problem is posed by the concentration of UCDs around the central regions of galaxy clusters (see Figure 7). If UCDs were formed by the tidal disruption of nucleated dwarf galaxies, they would still follow the same orbits and therefore have the same spatial distribution in the cluster as the undisrupted dwarf galaxies. Instead we find the UCDs are much more concentrated to the cluster centre. This suggests that their progenitors may be a primordial population that formed early around the central galaxies. This view is supported by Rakos \& Schombert (2004) who find that blue extragalactic globular cluster populations and nucleated dwarf elliptical galaxies form a single primordial sequence. As they point out, the connection between UCDs and this primordial population is very interesting. UCDs may be remnants of the original building blocks of central cluster galaxies.

\section{References}

Bassino, L. P., Muzzio, J. C., \& Rabolli, M. 1994, ApJ, 431, 634

Bekki, K., Couch, W. J., Drinkwater, M. J., \& Shioya, Y. 2003, MNRAS, 344, 399

Bekki, K., Couch, W. J., \& Drinkwater, M. J. 2001, ApJ, 522, L105

Binggeli, B., \& Cameron, L. M. 1991, A\&A, 252, 27

Bridges, T. J., Hanes, D. A., \& Harris, W. E. 1991, AJ, 101, 469

Djorgovski, S. G., et al. 1997, ApJL, 474, L19

Drinkwater, M. J., et al. 2000a, A\&A, 355, 900

Drinkwater, M. J., Jones, J. B., Gregg, M. D., \& Phillipps, S. 2000b, PASA, 17, 227

Drinkwater, M. J., et al. 2003, Natur, 423, 519

Durrell, P. R., et al. 2002, ApJ, 570, 119

Faber, S. M., \& Jackson, R. E. 1976, ApJ, 204, 668

Ford, H. C., Peng, E. W., \& Freeman, K. C. 2002, in The Dynamics, Structure \& History of Galaxies (astro-ph/0111035)

Gregg, M. D., \& West, M. J. 1998, Natur, 396, 549

Harris, W. E. 1996, AJ, 112, 1487

Hilker, M., Infante, L., Vieira, G., Kissler-Patig, M., \& Richtler, T. 1999, A\&AS, 134, 75

Hilker, M., Mieske, S., \& Infante, L. 2003, A\&A, 397, L9

Mieske, S., Hilker, M., \& Infante, L. 2002, A\&A, 383, 823

Mieske, S., Hilker, M., \& Infante, L. 2004, A\&A, in press

Moore, B. 2003, in Clusters of Galaxies: Probes of Cosmological Structure and Galaxy Evolution (astro-ph/0306596)

Phillipps, S., Drinkwater, M. J., Gregg, M. D., \& Jones, J. B. 2001, ApJ, 560, 201

Rakos, K., \& Schombert, J. 2004, AJ, 127, 1502

Shapley, H. 1938, BHarO, 908, 1 